\title{
Les business models de l'édition open source ; Le cas des logiciels
}

Open source editing business models; the case of software

\section{Amel Charleux et Anne Mione}

\section{OpenEdition}

\section{Journals}

Édition électronique

URL : http://journals.openedition.org/fcs/2088

DOI : $10.4000 /$ fcs. 2088

ISSN : 2261-5512

Éditeur

Association FCS

Référence électronique

Amel Charleux et Anne Mione, "Les business models de l'édition open source ; Le cas des logiciels ", Finance Contrôle Stratégie [En ligne], NS-1 | 2018, mis en ligne le 17 mai 2018, consulté le 17 mai 2018. URL : http://journals.openedition.org/fcs/2088; DOI : 10.4000/fcs.2088

Ce document a été généré automatiquement le 17 mai 2018

Tous droits réservés 


\title{
Les business models de l'édition open source ; Le cas des logiciels
}

Open source editing business models; the case of software

\author{
Amel Charleux et Anne Mione
}

Ce travail a bénéficié d'une aide de l'État gérée par l'Agence Nationale de la Recherche au titre du programme «Investissements d'Avenir » portant la référence ANR-10-LABX-11-01 (LabEx

Entreprendre)

\section{Introduction}

1 En 2016, 5,8 millions de contributeurs ont participé au développement de près de 19,5 millions de projets logiciels proposés sur des plateformes en open source $e^{1}$. Un telle ressource ne laisse pas indifférentes des entreprises aussi puissantes que Google, Amazon ou Facebook qui jouent le jeu de l'open source et proposent, elles aussi, certains de leurs logiciels sur ces plateformes. L'intérêt des géants n'est pas de pure forme. Il ne répond pas à une seule logique de veille concurrentielle et de contrôle du marché. Le secteur de l' open source est créateur d'emplois et de richesses. En France, il représente plus de 50000 emplois et 4 milliards d'euros de chiffre d'affaires ${ }^{1}$. Pourtant, la façon dont les acteurs de l'édition open source parviennent à créer la valeur ou à susciter la création en appelant la communauté à participer, puis à capturer la valeur créée n'est pas véritablement élucidée.

2 Pour montrer l'articulation entre la création et la capture de la valeur, le concept de business model (BM) s'avère utile. Le BM permet de penser la variété des façons dont les organisations peuvent créer, distribuer et capter la valeur (Lecocq et al., 2010). Il a précisément été forgé pour identifier de nouvelles formes, plus innovantes que la simple logique de constitution d'un avantage concurrentiel spécifique et de valorisation du droit de propriété intellectuelle. En ce sens, il constitue un instrument pertinent permettant de comprendre comment les nouvelles activités peuvent se réaliser. Dans le cas de l'open source, l'identification des BM est particulièrement intéressante parce qu'elle 
demande un effort particulier pour admettre de nouveaux modes de création de valeur, de considérer des formes alternatives de captation de valeurs créées collectivement et de concevoir l'articulation entre ces créations collectives et une appropriation individuelle. Le modèle de l'open source se fonde effectivement sur un mode original de création et d'appropriation de la valeur créée pour plusieurs raisons. En premier lieu, open source signifie « code source ouvert». Un éditeur qui propose un projet en open source ouvre les codes aux potentiels utilisateurs et contributeurs. Ceci a plusieurs conséquences. L'utilisateur peut télécharger le logiciel, le copier, le reproduire et, sous certaines conditions, le faire évoluer. La valeur créée par un contributeur bénéficie à l'ensemble de la communauté à laquelle il a accepté d'adhérer. La création de valeur par l'ouverture a été conceptualisée par le terme d'open innovation.

L'innovation ouverte définie par Chesbrough (2003) permet de concevoir l'intérêt que peut représenter l'ouverture dans la stimulation de l'innovation grâce aux apports des partenaires. Or, dans le cas de l'open source, cette ouverture présente la spécificité d'être extrême. Comme l'observent Chesbrough et al., (2006), l'open source innovation est un processus d'innovation encore plus ouvert que celui des consortiums classiques car l'ouverture est dirigée vers le plus grand nombre, sans sélection des contributeurs. Bien qu'existent des mécanismes de contrôle pour intégrer les contributions et faire évoluer le logiciel, le principe consiste à faire appel très largement aux communautés pour bénéficier de l'énergie et de la créativité collective. Le modèle de l'open source impose une conception particulière de la création de valeur par la multiplicité et le partage. Cette situation présente une originalité parce que l'entreprise qui s'engage dans cette voie n'a pas le choix de ses partenaires. Elle ne contrôle ni le nombre, ni le profil des contributeurs qui décident de rejoindre son projet. Dans ces conditions, la question de l'appropriation de la valeur créée se pose en des termes différents que dans le cas d'une innovation ouverte entre partenaires qui ont anticipé et négocié les conditions de partage de la valeur créée. Ouvrir les codes sources d'un logiciel aux contributeurs multiples permet de bénéficier de ressources supplémentaires. Pour autant, l'arbitrage pour l'ouverture ne se réalise-t-il pas au détriment de l'appropriation? L'ouverture du code s'accompagne-t-elle $\mathrm{du}$ renoncement à s'approprier individuellement la valeur collectivement créée? Comment les porteurs de projets arbitrent-ils le choix de l'ouverture relativement aux retours qu'ils anticipent de leur propre engagement dans le projet?

4 L'objectif de cette contribution est d'amener de la connaissance sur les façons dont les entreprises éditrices de logiciels créent la valeur et s'approprient la valeur créée dans le secteur de l'open source. Nous cherchons à comprendre comment, dans des conditions extrêmes d'innovation open source, elles parviennent à viabiliser leurs projets. L'analyse empirique des BM dans le secteur des logiciels, et notamment des logiciels open source, est de nature à contribuer aux questionnements théoriques associés à l'approche des BM en ce qu'elle amène une perspective originale sur la création et l'appropriation de la valeur. En effet, la perspective de l'open source requiert une conception nouvelle de la valeur qui amène à reconsidérer les raisonnements classiques en stratégie et donne un éclairage particulier à ceux de l'innovation ouverte. Certaines recherches ont identifié des BM dans le secteur open source mais elles ne problématisent pas véritablement l'articulation entre la valeur et l'appropriation. Finalement, le secteur open source invite à repenser la façon dont création et appropriation sont dépendantes et liées. Notre but est donc de décrire les 
BM adoptés par les éditeurs de logiciels dans le modèle open source et de montrer que ce secteur oblige à conceptualiser une forme originale de gestion de la valeur.

Pour ce faire, nous réalisons une étude approfondie du secteur des logiciels open source et nous proposons une taxonomie des BM de l'activité d'édition. La méthodologie mise en œuvre comprend une phase exploratoire qualitative et une phase quantitative. La phase exploratoire est constituée d'une revue documentaire, de l'analyse de sites internet, de comptes rendus de conférences et de 19 entretiens avec des professionnels. Cette approche permet de comprendre le fonctionnement du secteur et les modes de création de valeur. Ensuite, nous constituons une base de données de 199 logiciels libres et open source et nous mettons en œuvre la méthodologie quantitative descriptive pour créer une taxonomie (Baden-Fuller et Morgan, 2010; Borgès Da Silva, 2013).

La première partie définit le concept de BM et présente les spécificités du secteur de l' open source pour la création et l'appropriation de la valeur. Nous citons les travaux existants sur la question des BM dans le secteur et soulignons les précisions qu'elles nécessitent. La seconde partie présente la méthode de collecte et de traitement des données. La troisième partie expose les résultats de l'étude. Dans la partie discussion, nous revenons sur l'apport que constitue l'analyse empirique du secteur des logiciels open source à la compréhension des BM.

\section{Fondements théoriques}

\subsection{L'approche des BM dans le secteur de l'open source}

7 Le BM permet de poser en termes simples la question de la création et de l'appropriation de la valeur (Johnson et al., 2008). C'est un framework conceptuel qui permet d'organiser la création de valeur tout en garantissant sa rentabilité (Wirtz et al., 2016). Il s'agit de considérer l'interdépendance entre le BM et le modèle de revenus, posant respectivement les questions de la création de valeur et de sa captation (Zott et al., 2010). La définition de Teece (2010) questionne comment une entreprise délivre de la valeur aux consommateurs, les incite à payer pour cette valeur et convertit ces paiements en profits. En termes organisationnels, le BM décrit l'architecture organisationnelle et financière d'une affaire (Teece, 2010). Dans le secteur de l'open source, certaines spécificités amènent des questionnements particuliers du point de vue du BM et de l'articulation entre création et appropriation de valeur.

\subsubsection{Les spécificités du développement open source}

8 Le modèle de l'open source s'inscrit dans le courant de l'innovation ouverte (Chesbrough, 2003). Celui-ci bouleverse la conception traditionnelle de l'innovation en ouvrant les frontières de l'entreprise aux idées et aux acteurs extérieurs. Il ne s'agit plus d'être un first mover isolé (Lieberman et Montgomery, 1988) mais plutôt de développer une organisation capable d'innover collectivement. Cette nouvelle conception de l'innovation est en rupture avec les modèles classiques en ce qu'elle ouvre le champ des possibilités et stimule davantage l'innovation. Elle suggère donc une accélération de l'innovation qui pose la question de la viabilité marchande des idées nouvelles (Chesbrough, 2006; Teece, 2010). En effet, les protéger toutes a un coût alors que toutes ne sont pas susceptibles de convenir au marché ou au BM de l'entreprise (Chesbrough et Rosenbloom, 2002). 
L'innovation collective pose aussi la question du partage de l'innovation : l'implication de plusieurs acteurs dans le processus suggère qu'ils devront trouver les moyens de se partager la propriété et les fruits de l'innovation commune (West et al., 2014). L'innovation ouverte pose la question de la captation de valeur car il ne s'agit plus simplement de constituer un avantage compétitif, il s'agit aussi de trouver les moyens de l'exploiter (Chesbrough et Rosenbloom, 2002), ce qui amène naturellement le questionnement sur le BM. Ce questionnement est encore plus épineux dans le cas de l' open source (Chesbrough et al., 2006; Pénin, 2011).

9 Le modèle open source décrit une méthodologie de développement collectif qui peut s'appliquer à différents secteurs tels que le matériel informatique par exemple. Néanmoins, l'analyse du concept se réalise généralement par l'examen du secteur des logiciels informatiques qui constitue une illustration emblématique du fonctionnement du modèle. En 2003, Demil et Lecocq ont décrit l'industrie du logiciel en utilisant le prisme des droits de propriété, posant ainsi la question du partage de la valeur. Le schéma ci-dessous présente les différents modèles (Figure 1).

Figure 1. Représentation schématique de l'industrie du logiciel Inspirée du « Continuum des stratégies de droits de propriété » de Demil et Lecocq (2003)

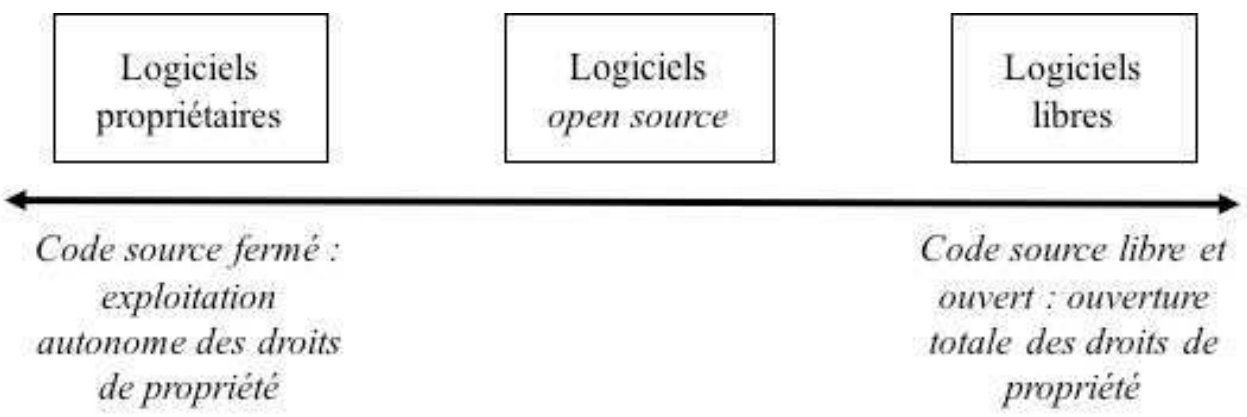

Les logiciels propriétaires se fondent sur la détention de droits de propriété intellectuelle privatifs et sur les avantages réservés à ce type de propriété. Les logiciels libres renoncent à ces droits tandis que le secteur de l'open source se situe entre les deux opposés du continuum. Les logiciels libres sont basés sur la logique d'un développement communautaire, libre et gratuit avec une ouverture maximale et une totale disponibilité des données. Le caractère communautaire tient à la nature variée des contributeurs : entreprises privées, collectivités, mais aussi et surtout développeurs particuliers intervenant en leur nom propre. Le caractère libre se fonde sur la liberté d'accéder, de modifier et de redistribuer les codes sources des logiciels à l'infini et ce, grâce à des licences spécifiques qui précisent les libertés accordées par les auteurs du logiciel aux utilisateurs (Muselli, 2008). Enfin, la gratuité est instituée aux deux niveaux de la création et de l'appropriation de la valeur. Le principe du volontariat s'applique aux contributeurs qui développent bénévolement le logiciel. Les utilisateurs de leur côté téléchargent et utilisent gratuitement le logiciel. Toutefois, le logiciel open source se situe sur une position intermédiaire entre le logiciel propriétaire et le logiciel libre. Cette position particulière tient à la possibilité de combiner les avantages du libre à des modèles commerciaux. En effet, un logiciel open source est un logiciel libre pour la création de valeur mais il peut autoriser une forme de redistribution commerciale voire, la fermeture. Finalement, les logiques à l'œuvre au long du continuum suggèrent_des BM différenciés. 
11 Ainsi, le secteur de l'open source pose le questionnement de la captation de valeur par ceux qui l'ont créée. Cette spécificité a provoqué l'intérêt des chercheurs qui ont identifié des BM originaux pour rendre compte de ces conditions extrêmes d'innovation open source. Dans les lignes suivantes, nous montrons que leurs apports méritent d'être complétés pour permettre une connaissance plus exhaustive et nuancée.

\subsection{Apports et limites des travaux sur les BM de l'open source}

De nombreuses recherches ont porté sur l'observation et l'étude des modèles open source (Benkeltoum, 2011; Chesbrough, 2010; Chesbrough et al., 2006). Elles contribuent à la connaissance empirique du secteur tout en enrichissant le cadre conceptuel sur les BM. Les chercheurs ont notamment montré les différentes positions des acteurs et la variété des BM liés. Du point de vue de l'entreprise focale par exemple, les contributeurs extérieurs peuvent être organisés sur le modèle de la communauté ou sur le modèle du marché (Boudreau et Lakhani, 2009). Chacune de ces deux organisations produit des BM différents. Ceux de la communauté sont basés sur le volontariat et la gratuité; ceux du marché sont compétitifs et basés sur le développement de produits et services complémentaires. Benkeltoum (2011) a montré que l'articulation entre création et appropriation de la valeur est originale dans le contexte de l'open source. En effet, l'organisation a un effet déterminant sur le processus même de création de valeur (Benkeltoum, 2011). Le caractère communautaire ou privé du porteur de projet a un effet sur l'attractivité des contributeurs et leur volonté de participer à l'élaboration du projet. Ainsi, les BM sont spécifiques car le signal envoyé par l'ouverture du projet influence la nature de la contribution, le profil des contributeurs et donc, les possibilités d'appropriation.

13 En termes organisationnels, l'organisation des communautés open source est similaire à celle des bazars orientaux, en opposition à la construction rigoureuse des cathédrales (Raymond, 1998). Néanmoins, malgré l'image qui en est donnée, le terme de "bazar» n'est pas synonyme de désordre et de chaos. Les communautés open source ne respectent pas un ordre conventionnel et peuvent apparaitre inorganisées en comparaison avec les structures plus classiques. Elles n'en sont pas moins régies par des rituels et des règles spécifiques. Les entreprises qui évoluent dans cet environnement ont d'ailleurs bien assimilé ce «bazar réorganisé » (Benkeltoum, 2011, p. 193). Pourtant, les travaux récents proposant une description des BM dans le champ du libre/open source ne permettent pas de saisir complètement cette articulation entre les éléments organisationnels qui relèvent de la création de valeur et ceux qui participent à sa captation.

Nous souhaitons mettre davantage en lumière le lien entre le déploiement d'un modèle d'innovation spécifique et l'adoption d'un système de revenu particulier (Zott et al., 2011). Pour ce faire, nous situons notre analyse au niveau du projet alors que le niveau d'analyse adopté dans la plupart des recherches porte sur l'entreprise. Ainsi, Lisein et al. (2009) mettent en évidence trois modèles adoptés par les sociétés évoluant dans le libre/open source: le modèle de la complexification qui repose sur la maitrise de la ressource humaine; le modèle du système clos qui repose sur la maitrise de la réputation avec un accès restreint aux données du logiciel et le modèle de l'intermédiation qui correspond à celui des passagers clandestins dont l'objectif est la maîtrise de la ressource technologique. Analysés sous l'angle de la théorie des ressources, ces BM permettent de comprendre des positionnements stratégiques en lien avec le système de valeurs du 
secteur. Reprenant ces travaux, Mouakhar et Tellier (2013) étendent la recherche à un échantillon d'entreprises plus important, composé de sociétés de services, et proposent une taxonomie de trois comportements open source en fonction de la légitimité des acteurs. Ils distinguent ainsi les sociétaires qui travaillent en étroite collaboration avec les communautés libres, les diplomates qui s'inscrivent dans les logiques libres sans pour autant développer d'échanges particuliers avec la communauté et enfin, les profiteurs qui jouent de la notoriété du libre mais qui n'hésitent pas à utiliser des logiciels propriétaires dans leurs solutions. Ces modèles sont basés sur l'adéquation des acteurs aux valeurs et à la philosophie du libre. Le BM est envisagé comme un outil stratégique qui permet d'approcher une représentation souvent abstraite de la structure de l'entreprise (Wirtz et al., 2016). Pourtant, cette conception est critiquée dans la littérature. Porter (2001) notamment souligne l'effet simplificateur qu'induisent les recherches sur le BM des entreprises, considérant que c'est une équation réductrice. Effectivement, l'approche du BM au niveau de l'entreprise est pertinente lorsque celle-ci ne porte qu'un seul projet. Dans ce cas, son activité ne repose que sur la viabilité de ce dernier. Or les entreprises peuvent constituer un portefeuille de projets au sujet desquels elles peuvent mettre en place des BM particuliers. L'originalité de notre contribution par rapport à la littérature tient à cette prise en compte du BM au niveau des projets logiciels. La méthodologie prend en compte cette perspective et s'attache à observer le BM des projets afin de saisir la complexité qui peut exister dans la construction d'une affaire (Casadesus-Masanell et Tarziján, 2012).

\section{Méthodologie}

La méthodologie comprend une phase exploratoire qualitative et une phase quantitative. La phase exploratoire permet de recueillir des données qualitatives primaires indispensables pour appréhender la complexité du terrain de recherche. La phase quantitative a pour objectif de mettre au jour les BM types utilisés par les entreprises du secteur pour l'édition de leurs logiciels.

\subsection{La phase exploratoire}

16 La phase exploratoire est réalisée grâce à une étude documentaire couplée à 19 entretiens ouverts. Les entretiens ont été réalisés auprès de professionnels du secteur pour comprendre la place des différents acteurs, leurs relations et leurs motivations quant au choix du modèle open source. Le profil des personnes interviewées comprend des managers, en charge des décisions stratégiques, et des ingénieurs informaticiens impliqués directement dans les processus de création de valeur. Cette phase a permis d'identifier les variables indispensables pour décrire les modes de création et de captation de la valeur.

\subsection{Constitution d'une base de données de logiciels open source}

17 Afin de proposer une taxonomie des BM utilisés par les éditeurs open source pour leurs projets, nous constituons une base de données de 350 logiciels, désignés par les sites spécialisés comme étant les plus utilisés par les intégrateurs informatiques. Nous procédons à une étude exhaustive des 350 logiciels afin d'éliminer ceux qui 
n'existent plus ou qui ne sont plus maintenus. Cette étape nous conduit à un échantillon final constitué de 199 logiciels actifs.

Le concept de BM est opérationnalisé selon la définition donnée par Teece (2010). Le BM est décrit comme la combinaison d'une architecture organisationnelle et financière. L'architecture organisationnelle est au service de la création de valeur et l'architecture financière est dédiée à la valorisation économique. Cette définition permet de saisir l'articulation entre ces deux éléments. L'architecture organisationnelle est définie ici par l'affiliation du projet - qui fait référence à la nature du porteur de projet - et par sa forme organisationnelle plus ou moins ouverte et structurée, décrite par la terminologie «bazar » ou « cathédrale » (Raymond, 1998). L'affiliation du projet est une variable clé du projet lui-même car elle joue un rôle dans le potentiel d'attrait ou de répulsion de la communauté. Certaines communautés refusent de contribuer à des projets affiliés à des entreprises commerciales. Le caractère commercial de l'affiliation influence dès lors directement les perspectives de création de valeur. L'architecture financière, pour sa part, est décrite selon les deux composantes de la propriété intellectuelle (PI) du logiciel et des sources de revenus. Le tableau ci-dessous présente cette opérationnalisation (Tableau 1).

Tableau 1. Le concept de business model

\begin{tabular}{lll}
\hline \multirow{2}{*}{$\begin{array}{c}\text { Business model } \\
\text { (Teece, 2010) }\end{array}$} & Architecture organisationnelle & Affiliation \\
\cline { 2 - 3 } & Architecture financière & Forme organisationnelle \\
\cline { 2 - 3 } & Propriété intellectuelle \\
\hline
\end{tabular}

La composition des variables est décrite dans la figure ci-après (Figure 2).

Figure 2. Les variables du modèle

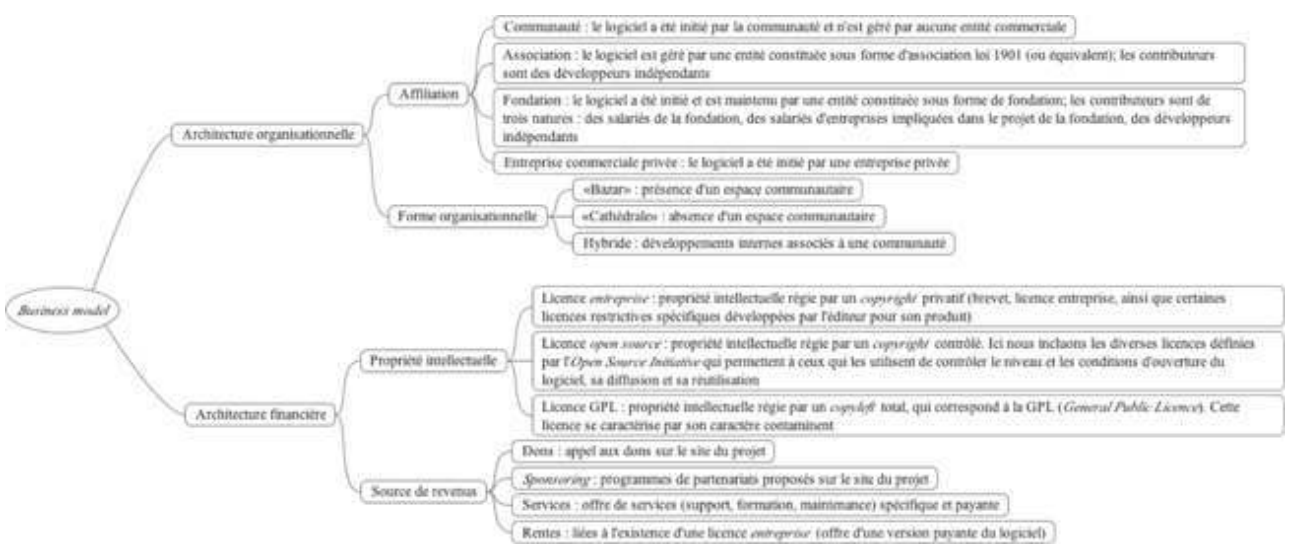

Des variables supplémentaires sont considérées comme illustratives (Tableau 2): la nature du logiciel - qui permet de qualifier le processus d'innovation - est définie par le statut du code (ouvert ou fermé), la gouvernance de l'éditeur selon la typologie de Demil et Lecocq (2006) inspirée des travaux de Williamson (1996) et l'organisation de l'écosystème au sens de Boudreau et Lakhani (2009). 
Tableau 2. Les variables illustratives du modèle

\begin{tabular}{ll}
\hline & Ouvert, si les codes sources sont téléchargeables gratuitement \\
\cline { 2 - 3 } Nature du logiciel & $\begin{array}{l}\text { Fermé, si les codes sources sont soumis à souscription d'un forfait } \\
\text { ou achat d'une licence }\end{array}$
\end{tabular}

\begin{tabular}{ll}
\hline & $\begin{array}{l}\text { Marché, lorsque l'écosystème est compétitif, avec des acteurs qui } \\
\text { vont développer des produits et services complémentaires } \\
\text { spécifiques }\end{array}$ \\
\cline { 2 - 2 } $\begin{array}{l}\text { Organisation de l'écosystème de } \\
\text { l'acteur }\end{array}$ & $\begin{array}{l}\text { Communauté, lorsque l'écosystème est basé sur le volontariat, } \\
\text { l'échange et la gratuité }\end{array}$ \\
\hline
\end{tabular}

Hiérarchique, gouvernance gérée par des contrats d'employabilité, des incitations à la contribution faibles et un contrôle fort

Network, gouvernance relationnelle, basée sur l'échange et la réciprocité. Les incitations à la contribution sont moyennes, de même que l'intensité du contrôle

Modèle de gouvernance

Marché, gouvernance contractuelle et basée sur un niveau d'incitation à la contribution fort de par l'aspect compétitif de l'organisation, et un faible contrôle

Bazar, gouvernance qui repose sur les licences ouvertes et le copyleft. Le produit est au centre de la relation avec cependant des incitations à la contribution et un niveau de contrôle faibles

21 La base de données est complétée par la catégorie à laquelle appartient le logiciel (Tableau 3), le nom de la/les licence(s) utilisée(s), l'année de création du logiciel, le nombre de lignes de code, le nombre de contributeurs au logiciel, le nombre d'utilisateurs ainsi que le langage de programmation utilisé.

22 L'ensemble de ces variables est renseigné par une recherche documentaire des données secondaires collectées sur les divers sites internet des projets (sites institutionnels, Wikipédia, plateformes de développement), sur des blogs et des articles de presse. 
Tableau 3. Les catégories de logiciels

\begin{tabular}{|c|c|c|}
\hline Accélérateur HTTP & Cloud Computing & Contrôle des postes à distance \\
\hline Déploiement et sauvegarde & Gestion de parc & Haute disponibilité \\
\hline Sécurité & Réseaux privés virtuels & Firewall \\
\hline Supervision et métrologie & SE Linux \& BSD & Virtualisation \\
\hline VoIP / Téléphonie & $\begin{array}{c}\text { Messagerie, e-mainling \& } \\
\text { groupeware }\end{array}$ & Autres \\
\hline \multicolumn{3}{|c|}{ Développement et couches intermédiaires } \\
\hline Annuaire d'entreprise & Bases de données & Big Data \\
\hline BPM / Workflow & Outils de développement & Tests \& Intégration continue \\
\hline Entreprise service bus & Bibliothèques & Frameworks mobiles \\
\hline $\begin{array}{l}\text { Authentification, fédération et } \\
\text { gestion d'identité }\end{array}$ & Outils de test de charge & Infrastructure à clés publiques \\
\hline Extract Transform Load & $\begin{array}{l}\text { Serveur HTTP et serveurs } \\
\text { d'application }\end{array}$ & Moteurs de recherche \\
\hline \multicolumn{3}{|l|}{ Autres } \\
\hline \multicolumn{3}{|l|}{ Applications } \\
\hline Customer Relation Management & Décisionnel : reporting & Containt Management System \\
\hline E-commerce & Entreprise Ressource Planning & Portail \\
\hline GED \& EDM & Bibliothèque et documentation & e-Learning \\
\hline Réseaux sociaux d'entreprise & Blogs, Wiki et forums & $\begin{array}{l}\text { Product Information } \\
\text { Management }\end{array}$ \\
\hline Suivi d'audience & Outils de partage de documents & Digital Asset Management \\
\hline Décisionnel & Autres & \\
\hline
\end{tabular}

\subsection{Traitements statistiques} Utilisée dans la recherche en gestion (Malhotra et Majchrzak, 2014; Okazaki, 2006), cette méthode est recommandée dans le traitement de données catégorielles, multinomiales, indépendantes et sans nombre de groupes définis à priori. Elle permet de révéler des groupements naturels dans des bases de données volumineuses (Tkaczynski, 2017). Nous avons testé l'hypothèse de nullité et l'indépendance des variables au moyen du khi-deux. Les résultats invalident la première hypothèse de nullité et corroborent la seconde hypothèse de dépendance des variables ${ }^{1}$. Ainsi par exemple, la politique de licence adoptée pour le logiciel a un impact sur le modèle d'organisation des contributions, mais aussi sur les sources de revenus. Le BM correspond à une démarche globale où les choix adoptés pour chaque paramètre influencent l'ensemble du modèle. Malgré cette dépendance des variables, la méthode des TwoStep Cluster permet de procéder à la manipulation car elle est " assez résistante aux violations des hypothèses d'indépendance »"

Pour limiter les biais liés au tri, nous réalisons l'analyse du fichier de façon linéaire et incrémentale. Ainsi, l'ordre dans lequel les observations sont rentrées n'a pas d'incidence sur les résultats. Aucun classement hiérarchique des variables et des composantes n'est réalisé. Les observations sont saisies de façon aléatoire et nous avons procédé à plusieurs tests afin de vérifier la stabilité du nombre de clusters finaux.

Nous réalisons deux études successives appliquant l'analyse TwoStep Cluster. Une première étude met en évidence trois groupes avec une qualité correcte des clusters. Une 
recherche supplémentaire fait apparaitre quatre groupes et apporte une qualité supérieure aux clusters. La figure suivante (Figure 3) présente la qualité de l'analyse.

Figure 3. Qualité et résumé du TwoStep Cluster

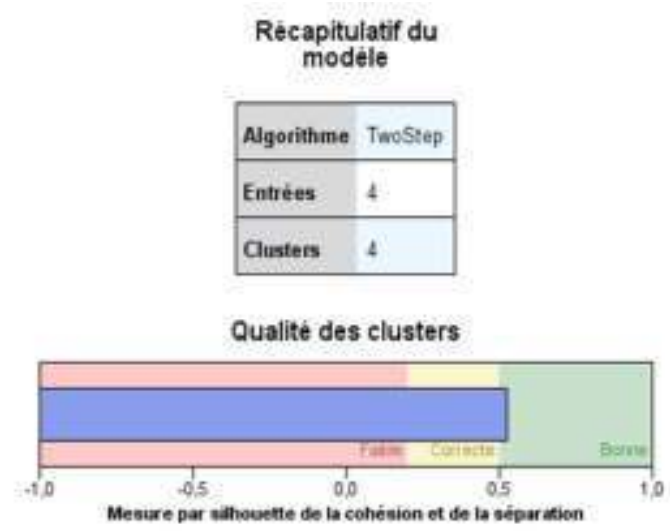

\section{Résultats}

L'analyse permet de décrire quatre groupes naturels, homogènes et différenciés (Tableau 4).

Tableau 4. Quatre BM naturels, homogènes et différenciés

\begin{tabular}{|c|c|c|c|c|}
\hline & 1 & 2 & 3 & 4 \\
\hline $\begin{array}{c}\text { Statut } \\
\text { juridique }\end{array}$ & $\underset{(56 \%)}{\text { Entreprise privée }}$ & $\begin{array}{l}\text { Entreprise privée } e^{(43.1 \%)} \\
\text { Communauté }{ }^{(25.5 \%)}\end{array}$ & $\begin{array}{c}\text { Fondation }{ }^{(90,9 \%)} \\
\text { Communaute } \\
(29.8 \%)\end{array}$ & $\begin{array}{c}\text { Association }^{(90 \%)} \\
\text { Communauté }^{(44,7 \%)}\end{array}$ \\
\hline $\begin{array}{c}\text { Forme } \\
\text { orga. }\end{array}$ & Hybride ${ }^{(62 \%)}$ & $\begin{array}{c}\text { Hybride }^{(34.8 \%)} \\
\text { Bazar }^{(25.5 \%)}\end{array}$ & Bazar ${ }^{(41.2 \%)}$ & Bazar $^{(31.4 \%)}$ \\
\hline PI & Hybride ${ }^{(98.4 \%)}$ & $\begin{array}{c}\mathrm{GPL}^{(45 \%)} \\
\text { Open source }^{(41.6 \%)}\end{array}$ & Open source $e^{(58,4 \%)}$ & GPL ${ }^{(55 \%)}$ \\
\hline $\begin{array}{l}\text { Source de } \\
\text { revenus }\end{array}$ & $\begin{array}{c}\text { Rente et autres } \\
(100 \%)\end{array}$ & $\begin{array}{l}\text { Services }{ }^{(90,7 \%)} \\
\text { Services + sponsoring }^{(87.5 \%)} \\
\text { Sponsoring } \\
(86,7 \%)\end{array}$ & $\begin{array}{c}\text { Dons + sponsoring } \\
\text { Dons }^{(31.7 \%, 3 \%)}\end{array}$ & Dons ${ }^{(68.3 \%)}$ \\
\hline
\end{tabular}

Le premier groupe comprend les logiciels affiliés à des entreprises privées commerciales dont la politique de propriété intellectuelle est mixte : licence ouverte combinée à une licence entreprise fermée. Le logiciel génère des revenus qui proviennent essentiellement de l'exploitation de la licence entreprise combinée aux autres sources possibles que sont le service et le sponsoring. L'organisation des divers flux d'information liés au projet se font aussi bien sous la forme de bazar (existence d'une communauté) que sous la forme de cathédrale (pour les flux liés à la version entreprise). Le deuxième groupe est constitué également de logiciels affiliés à des entreprises privées mais aussi de logiciels initiés par des communautés. Contrairement au premier groupe, la politique de PI appliquée aux projets est exclusivement ouverte (GPL et autres open source). Les revenus tirés des logiciels de ce groupe proviennent essentiellement des offres de services et du sponsoring. L'organisation des flux d'information et de contribution est quant à elle hybride avec une dominance de la structure en bazar. Le groupe 3 est majoritairement représenté par les logiciels portés par des fondations. La politique de licence adoptée dans ce groupe est 
exclusivement open source. Les revenus sont générés essentiellement par le couple dons et sponsoring. Le groupe 4 est, pour sa part, constitué de logiciels initiés par des communautés et des associations. Contrairement au groupe 3, la licence appliquée est uniquement la GPL et les sources de revenus sont fortement liées aux dons purs.

\subsection{Quatre BM pour l'édition de logiciels}

L'analyse descriptive des 199 logiciels permet d'identifier quatre types de BM d'édition de logiciels (Tableau 5). Nous proposons de dissocier le BM qui est propre aux projets libres et que nous qualifions de BM de l'engagement des autres logiciels développés en open source qui renvoient à une logique hybride permettant de combiner création de valeur et capture marchande de la valeur créée. Nous qualifions les trois modèles open source de BM de l'exploration, de l'expertise ou de l'optimisation.

Tableau 5. Taxonomie des BMs de l'édition

\begin{tabular}{|c|c|c|c|c|c|}
\hline & \multicolumn{4}{|c|}{ Inscription marché } \\
\hline & & \multicolumn{4}{|c|}{ Stratégie d'ouverture } \\
\hline & & \multicolumn{3}{|c|}{ LOGICIELS OPEN SOURCE } & \multirow[b]{2}{*}{ ENGAGEMENT } \\
\hline & & OPTIMISATION & EXPERTISE & EXPLORATION & \\
\hline \multirow{2}{*}{$\begin{array}{l}\text { Architecture } \\
\text { organisationnelle }\end{array}$} & Statut juridique & Entreprise privée & Entreprise privée & Fondation & $\begin{array}{c}\text { Organisation à but } \\
\text { non lucratif }\end{array}$ \\
\hline & $\begin{array}{l}\text { Forme } \\
\text { organisationnelle }\end{array}$ & $\begin{array}{c}\text { Hybride } \\
\text { (Cathédrale et } \\
\text { Bazar) }\end{array}$ & Hybride à bazar & Bazar & Bazar \\
\hline \multirow{2}{*}{$\begin{array}{l}\text { Architecture } \\
\text { financière }\end{array}$} & $\begin{array}{l}\text { Propriété } \\
\text { intellectuelle }\end{array}$ & $\begin{array}{c}\text { Copyright }+ \\
\text { Copyleft }\end{array}$ & Copyleft contrôlé & Copyleft contrôlé & Copyleft total \\
\hline & Source de revenus & $\begin{array}{l}\text { Rente + Service + } \\
\quad \text { Sponsoring }\end{array}$ & Service & Dons + Sponsoring & Dons \\
\hline Produit & Statut du logiciel & $\begin{array}{c}\text { Hybride } \\
\text { (ouvert et fermé) }\end{array}$ & Ouvert & Ouvert & Ouvert \\
\hline Firme & $\begin{array}{l}\text { Modèle de } \\
\text { gouvernance }\end{array}$ & $\begin{array}{c}\text { Hiérarchique + } \\
\text { Network }\end{array}$ & $\begin{array}{c}\text { Hiérarchique }+ \\
\text { Bazar }\end{array}$ & Network & Bazar \\
\hline Écosystème & $\begin{array}{l}\text { Organisation de } \\
\text { l'écosystème }\end{array}$ & $\begin{array}{l}\text { Marché + } \\
\text { Communauté }\end{array}$ & $\begin{array}{c}\text { Marché + } \\
\text { Communauté }\end{array}$ & $\begin{array}{c}\text { Marché + } \\
\text { Communauté }\end{array}$ & Communauté \\
\hline
\end{tabular}

\subsubsection{Le BM de l'engagement}

Ce BM concerne les projets logiciels libres au sens de free software - «logiciels libres et gratuits ». Les projets sont portés par des organisations à but non lucratif (associations, communautés ou aucune forme) qui n'adoptent pas de logique commerciale. Elles peuvent toutefois recueillir des financements qui permettent d'assurer les frais de fonctionnement liés aux projets (serveurs, salons éventuels... etc.). Les communautés sont organisées en bazar. Les contributions sont libres et bénévoles, basées sur le principe du strict copyleft ; la relation entre les acteurs est très peu hiérarchisée et elle est orientée "produit »; les incitations à la collaboration sont faibles et le niveau de contrôle est également réduit ce qui octroie une grande liberté dans la participation aux projets et à la création de valeur. La captation de la valeur est occasionnelle et non organisée ; certains contributeurs participent financièrement à l'organisation d'événements de promotion du logiciel sous forme de donation et les frais de fonctionnement sont assumés par les membres de façon arbitraire, volontaire et non systématique. Ce BM se positionne hors du marché et convient pour le développement de projets qui ont une forte orientation 
politique ou philosophique. Les initiateurs sont des personnalités engagées pour une cause qui excluent par principe les objectifs économiques. Ce mode de fonctionnement crée une forte attractivité et suscite une grande adhésion des contributeurs. La popularité et le succès de certains logiciels renommés sont dus à ce modèle de l'engagement qui caractérise le secteur du logiciel libre.

\subsubsection{Le BM de l'exploration}

Ce BM concerne des logiciels développés essentiellement par des fondations. Les projets sont soutenus par un réseau fort de partenaires qui s'impliquent financièrement mais également en termes de mise à disposition de ressources. La gouvernance correspond au modèle du network avec des relations basées essentiellement sur l'échange et la réciprocité des incitations. Ces dernières sont d'intensité moyenne: les meilleurs développeurs peuvent être embauchés par les partenaires, invités à des événements ou devenir ambassadeurs; les partenaires professionnels bénéficient ici aussi d'un système de valorisation de la contribution qui débouche sur de la certification type « expert » et une meilleure visibilité sur le site du projet - visibilité silver, gold ou premium-.

31 Les préoccupations financières liées aux projets d'exploration concernent la viabilisation et le maintien de la forme organisationnelle du bazar: la communauté est libre de contribuer au code source du logiciel, sans sélection à l'entrée. Pour ce qui est de la contribution des partenaires, un contrôle interne important est instauré afin de garantir la qualité et la stabilité du logiciel. Celui-ci est proposé sous licence open source (type copyright contrôlé). Les revenus générés par le projet sont issus de divers dons mais aussi et surtout d'un programme de sponsoring élaboré: les partenaires s'engagent avec des enveloppes qui peuvent être très élevées et des ressources propres (pour le projet OpenStack par exemple, le partenariat Platinium est à 500000 dollars/an avec un engagement de mise à disposition de deux développeurs à plein temps dédiés au projet de la fondation). Les fondations portent dans leur grande majorité des projets à caractère technique très innovant. Leur objectif est donc le développement technologique et la recherche. Elles naissent à la faveur de projets que l'on pourrait qualifier d'exploratoires et tiennent à se maintenir hors des contraintes du marché (notamment des contraintes d'exploitation pures), afin de préserver une grande liberté d'innovation technique. Les éditeurs qui adoptent ce BM pour leurs projets privilégient ainsi la création de valeur en récupérant toutefois certains bénéfices tels que la possibilité de repérer des talents, de recruter des experts, de communiquer de façon efficace et ciblée sur le logiciel ou encore de bénéficier de la valeur créée et de la faire croître en investissant dans des infrastructures qui permettent de maintenir un environnement de recherche qualitatif.

\subsubsection{Le BM de l'expertise}

Le BM de l'expertise concerne les logiciels affiliés à une structure privée qui capitaliserait sur son savoir-faire lié au développement et non sur la rente qu'elle pourrait dégager de la vente des logiciels. Ainsi, le produit de ces entreprises est accessible et ouvert sous des licences open source (type copyright contrôlé). L'ensemble de la communauté peut y participer de façon libre mais encadrée par des road maps et des comités de pilotage. Le modèle organisationnel s'inspire massivement du bazar, tout en y insufflant un certain cadre essentiellement lié aux orientations de développement souhaitées par l'entreprise. Contrairement au modèle de l'exploration, les logiciels développés sous le modèle de 
l'expertise doivent rester proches des besoins du marché et répondre autant que possible à des demandes clients. Cette organisation en bazar hybride permet à l'entreprise d'augmenter sa base d'utilisateurs. Les rendements croissants d'adoption, très sensibles dans ce secteur (Arthur, 1989), jouent favorablement pour la diffusion du logiciel. Au-delà des contributeurs indépendants, ces rendements sont susceptibles de convaincre les entreprises d'adopter les nouveaux logiciels. L'image du logiciel, son caractère innovant, son professionnalisme ou sa qualité bénéficient à l'éditeur qui renforce ainsi son statut d'expert. La stratégie d'ouverture du code sert ici la création de valeur dans le gain de spécialisation et d'expertise.

\subsubsection{Le BM de l'optimisation} core ». Cette catégorie concerne exclusivement des logiciels proposés en deux versions : une version communautaire gratuite pour le core du logiciel (ou le cœur) et une version fermée et payante pour toutes les parties périphériques. La première version est proposée sous une licence open source (de type copyright contrôlé) et la seconde version améliorée est éditée sous une licence appelée entreprise (de type copyright privatif). Cette version repose largement sur la version ouverte mais n'est maintenue que par les développeurs de la société éditrice sous le modèle d'organisation de la cathédrale. La gratuité de la première version est entretenue par la rente dégagée par la version payante. Ce modèle à deux vitesses conquiert de plus en plus d'éditeurs et se trouve à la croisée des modèles propriétaire et libre. Les acteurs qui adoptent ce BM de développement logiciel cherchent à optimiser leurs investissements en capitalisant tout à la fois sur l'innovation et l'inventivité de la communauté, et sur la rigueur et la sécurité monnayable d'une version entreprise. Autour du logiciel se développe également toute une panoplie de services professionnels qui permettent de dégager des revenus importants car l'expertise sur le logiciel est forte et difficilement imitable.

Par ailleurs, ce BM impose une gouvernance hybride car la création de valeur doit être stimulée par l'ouverture et gérée par le contrôle. Le logiciel doit évoluer par les contributions multiples. Les relations avec la communauté sont établies sous forme de network où la créativité est autorisée bien qu'encadrée (contributions orientées grâce à une road map et un comité de pilotage). Des incitations à la contribution sont proposées : les développeurs indépendants impliqués fortement peuvent avoir des possibilités d'embauche par l'éditeur, des invitations à des événements, des conférences... etc.; les autres contributeurs que sont les entreprises partenaires (souvent des Entreprises de Services du Numérique (ESN), anciennement Sociétés de services en ingénierie informatique (SSII)) bénéficient d'un système de points où chaque contribution et/ou chaque vente de licence est valorisée et donne lieu à une meilleure visibilité sur le site internet du logiciel - visibilité silver, gold ou platinium. Le logiciel doit être stabilisé en interne avec les contributions des salariés encadrés par des relations hiérarchiques traditionnelles. Ces contributions sont fortement contrôlées - la création de valeur est orientée - sans incitations supplémentaires.

Enfin, l'écosystème dans lequel évolue et se développe le projet est également « hybride » car la relation de l'éditeur avec la communauté est basée sur le volontariat et la gratuité de la contribution et, en même temps, sur des relations de partenariat contractuelles avec les autres contributeurs que sont les intégrateurs de la solution (les ESN). 


\subsection{L'articulation entre création et appropriation gérée par l'ouverture et l'orientation marché}

36 L'identification des différents BM peut être lue dans l'articulation entre les deux mouvements contradictoires que sont l'ouverture du logiciel et l'inscription dans le marché (Tableau 5). Cette description rend compte de la complexité qui est à gérer. À gauche se situent les logiciels qui présentent une inscription forte dans le marché avec une ouverture limitée. À droite sont positionnés les logiciels très ouverts qui s'inscrivent peu dans la recherche de bénéfices liés au marché. Cette représentation met en évidence une tension entre ces deux orientations et finalement la difficulté de les articuler.

Cette tension amène à considérer la signification de la valeur. L'ouverture du logiciel est réalisée pour produire une valeur liée au développement du logiciel. En ouvrant les codes, le porteur du projet attend une contribution importante de la communauté. La valeur créée par cette ouverture peut être l'enrichissement du logiciel par des évolutions dans les performances, la rapidité, la fiabilité, le confort. La création de valeur peut également recouvrer la créativité. Par exemple, les contributions peuvent révéler des adaptations du logiciel à des secteurs ou à des usages inattendus dans des conditions particulières, par des lead users originaux. La diversité des contributeurs et leur multiplicité est de nature à enrichir le spectre des usages et à inspirer des évolutions plus imaginatives et créatives que si elles n'étaient portées que par les développeurs en interne. Également, le fait d'ouvrir les codes permet de tester l'attrait du logiciel et sa pertinence pour le marché. Enfin, l'ouverture du code favorise la diffusion qui est au bénéfice de la conquête du marché.

38 Au-delà de cette valeur liée au logiciel lui-même, la décision d'ouvrir les codes signale l'adhésion à des principes communautaires. Ils sont parfois adoptés par les entreprises privées qui arbitrent entre les bénéfices de l'ouvert et du fermé. Les contributeurs lisent ces stratégies et décident ou non d'y participer.

Le caractère hybride concerne aussi les modes de gouvernance en direction de la communauté et des salariés et les parts commercialisées ou laissées libres. Finalement, l'articulation entre création et appropriation de la valeur créée se réalise par un management subtil qui tente de dépasser les contradictions liées à la capture individuelle d'une création collective. Par exemple, dans le modèle de l'optimisation, l'éditeur peut proposer une même solution en version gratuite ou à la vente avec des parties protégées par une politique nuancée de copyright. Cette finesse est nécessaire car la politique de copyright joue en amont sur l'attractivité des contributeurs. Les mouvements de fermeture ne doivent pas nuire à la contribution communautaire, sous peine de voir les logiciels délaissés par les développeurs. Les entreprises qui choisissent le BM de l'expertise pour leur offre de logiciel proposent des services professionnels sophistiqués qui reposent en grande partie sur l'expertise qu'ils acquièrent grâce à ce processus itératif d'allers-retours entre la communauté et les clients. Ce positionnement se nourrit de la combinaison des avantages de l'ouvert et du fermé: en contribuant au développement du logiciel, l'éditeur prend part à l'innovation et bénéficie de l'apport de la communauté. L'expérience acquise et l'expertise reconnue dans cette confrontation aux autres peuvent être réinvesties dans des prestations rémunérées dans lesquelles l'éditeur adopte le statut de fournisseur de solutions vis-à-vis du client. Les compétences acquises dans cette relation spécifique ne sont pas nécessairement partagées. Au 
contraire, elles peuvent être gardées secrètes et donner une spécificité et donc une idiosyncrasie qui distingue l'éditeur de ses concurrents. Les BM de l'optimisation et de l'expertise sont donc le choix des éditeurs qui développent leurs projets au plus proche des attentes des clients et qui savent néanmoins jouer le jeu de la communauté en arbitrant entre ce qu'ils donnent et les bénéfices qu'ils peuvent en retirer.

\section{Discussion} lieu, nous proposons une contribution pratique, servant une meilleure identification et compréhension des BM d'édition dans le secteur du logiciel libre et open source. Cette approche empirique permet de donner corps à un concept en développement et dont la définition n'est pas encore circonscrite (Wirtz et al., 2016; Zott et al., 2010). Dans l'effervescence des nouvelles pratiques, nous contribuons à apporter une clarification. En effet, malgré l'intérêt suscité par ce phénomène, les recherches menées ne permettent pas une compréhension réelle de la façon dont les différents participants au secteur mènent leurs activités. Une première contribution est de situer les positions des intervenants au sein de l'industrie. En effet, les recherches portant sur ce sujet abordent le secteur dans sa globalité, sans opérer de distinction claire entre les entreprises positionnées différemment sur la chaîne de valeur. Ainsi, les éditeurs de logiciels sont agglomérés aux sociétés de services dans un ensemble commun, alors même que leurs activités sont différentes. Les éditeurs sont à l'origine des logiciels. Ce sont les porteurs des projets. Il leur appartient de réaliser les choix stratégiques liés aux questions de création et de captation de valeur et de gérer l'articulation entre ouverture et protection. Les sociétés de services (ESN), pour leur grande part, occupent la place d'intermédiaires entre les éditeurs et les clients. Elles peuvent contribuer à des solutions logicielles mais ne les portent pas. Dès lors, le BM de ces entreprises est fondamentalement différent de celui des éditeurs. Dans notre contribution, nous mettons en lumière la spécificité de la position de l'éditeur et des négociations stratégiques auxquelles il est confronté. Également, les modes libre et open source sont souvent assimilés sous le vocable générique de FLOSS (Free-Libre Open Source Software). À notre sens, ces amalgames induisent de la confusion et nuisent à la compréhension des enjeux stratégiques des différents intervenants du secteur. Notre second apport est donc une contribution à la connaissance et à la distinction des modes libres ou open source et des enjeux stratégiques qui leur sont liés.

41 Au niveau théorique, nous souhaitons contribuer à une réflexion sur les BM. Le concept se développe traditionnellement dans l'identification de nouvelles activités. En effet, la terminologie de BM a mobilisé la communauté scientifique dès les années 90 dans le champs du e-commerce (Lecocq et al., 2010; Zott et Amit, 2010). Ce sont ensuite les entreprises de la nouvelle économie numérique qui ont été les plus innovantes dans la recherche de solutions économiques permettant de profiter du nouveau marché que représentait l'Internet (Ghaziani et Ventresca, 2005; Magretta, 2002) et ont intéressé les chercheurs. Très spécialisée au départ, la recherche sur les BM a trouvé tout son sens et toute son ampleur actuelle dans les nouvelles pratiques d'innovation ouverte (Chesbrough, 2003) et concerne toutes les entreprises qui sont amenées à innover dans des conditions nouvelles. Or, une des questions posées par l'adoption du concept de BM porte sur le niveau de précision choisi pour expliquer les façons de constituer la valeur et 
de la capter. Alors que le concept se situe à un niveau relativement global (Rajala et al., 2003), nous avons proposé d'entrer dans un niveau de précision plus fin pour bien saisir la complexité des BM. Une de ces complexités réside dans l'unité d'analyse choisie. Alors que la recherche sur ce thème s'est largement concentrée sur l'entreprise (Bonaccorsi et al., 2006; Chesbrough, 2010; Wirtz et al., 2010), certains chercheurs abordent la question du point de vue de la combinaison de plusieurs BM dans une même entreprise (Casadesus-Masanell et Tarziján, 2012). Le niveau d'analyse se déporte alors vers le projet ou l'offre. Dans cette recherche, en prenant pour unité d'analyse le logiciel, nous développons l'idée d'un portefeuille de BM pour les éditeurs. En fonction du degré de préoccupation "philosophique» (valeurs du libre) combiné à un degré de préoccupation économique, nous décrivons quatre BM de projets que nous articulons grâce aux deux composantes de création et de capture de la valeur. Nous enrichissons notre modèle de variables liées au réseau qui sont également importantes dans le concept (Wirtz et al., 2016). Nos résultats montrent notamment que lorsque l'éditeur souhaite s'investir dans le développement d'un logiciel dont le degré d'innovation est important ou qu'il souhaite explorer de nouvelles perspectives, il opte pour le BM de l'exploration. Il choisit préférentiellement d'affilier le projet à une fondation, de travailler étroitement avec la communauté et garde le code source ouvert afin de s'assurer la contribution de la communauté. Pour pérenniser son activité et dégager des revenus, il peut mettre en place un système de recueil de dons adossé à un programme de partenariats. À la question de recherche initiale " comment, dans des conditions extrêmes d'innovation open source, les entreprises parviennent-elles à viabiliser leurs projets? », nous apportons une réponse par la description des BM.

Nous tenons à souligner que pour certains BM, les caractéristiques ne sont pas de nature unique mais peuvent présenter des hybridations. En effet, nous observons une porosité et une dynamique entre les trois idéaux-types de l'open source. Cette hybridation et cette flexibilité constituent un élément de réponse concernant la viabilité du projet. En réalité, le projet peut être nourri puis gagner une forme d'autonomie puis se réapprovisionner auprès de la communauté dans la mesure où un équilibre est trouvé pour les différents contributeurs. Au sein de ce processus, des préoccupations marchandes pures auront du mal à agréger les bonnes volontés des contributeurs qui sont attachés aux valeurs de partage et de gratuité. Mais les dilemmes ne sont pas toujours radicaux et les registres peuvent varier entre les deux extrémités des valeurs associées aux ordres civique ou marchand. Il faut également intégrer les valeurs associées au projet et considérer l'opportunité que représente l'accès au réseau pour les développeurs passionnés.

Cette perspective ouvre une réflexion sur la dimension ontologique du concept d'open source qui constitue un questionnement rémanent de l'industrie du logiciel. Il s'inscrit, en effet, dans une philosophie politique associée à des principes de coopération, de partage et de résistance au modèle marchand dominant. Il s'agit d'une véritable quête, avec des valeurs revendiquées et la proposition d'un modèle alternatif d'organisation sociétale. Dans cette perspective, la notion de BM prend une signification particulière. Cette notion qui n'est pas nécessairement citée en tant que telle répond d'une authentique préoccupation des acteurs qui se sont immédiatement interrogés sur les conditions de mise en œuvre et de viabilité du système du libre. Le concept de BM trouve donc dans ce secteur une dimension idéale en ouvrant la voie à de nouvelles façons de produire et d'échanger dans des formes viables et pérennes. Il se libère ainsi d'une forme 
pragmatique (combien cela fait gagner?) pour une forme plus idéalisée (comment cela peut-il fonctionner?).

En termes de limites et de voies futures de recherches, nous souhaitons souligner l'intérêt que présenteraient des données strictement financières qui permettraient une évaluation plus précise des revenus. Elles ne sont pas toujours disponibles, ni accessibles : certains projets d'origine étrangère n'ont pas d'obligations de publication de résultats ; lorsque les chiffres existent, ils portent sur les entreprises et non sur les projets qui constituent notre unité d'analyse et enfin, une partie importante des logiciels est développée par des communautés sans structure légale. Des études de cas approfondies pourraient permettre d'apporter une réponse plus argumentée sur la question de la rentabilité et de la valorisation des actifs. La direction future de nos travaux porte sur les portefeuilles de projets, la compatibilité des BM associés, leur hybridation et la dynamique des BM.

\section{Conclusion}

Auréolé de valeurs de partage et de liberté, le secteur de l'open source suscite l'intérêt des professionnels et des chercheurs. Certaines entreprises s'engagent résolument tandis que d'autres "misent pour voir", afin de ne pas se laisser distancer par cette opportunité d'un mode alternatif de création de valeur. Les chercheurs, pour leur part, admettent le bouleversement de paradigme et tentent de donner les clés permettant de comprendre ce phénomène. Notre contribution s'inscrit dans ce sens et nous apportons une clarification en proposant de décrire les différents acteurs du secteur, en nous centrant sur le rôle clé des éditeurs. Les éditeurs génèrent des projets et choisissent le mode de développement en ouvrant ou limitant l'accès aux codes sources. Leurs objectifs stratégiques peuvent s'opposer ou s'inscrire dans une logique marchande. Nous tenons compte de ces deux orientations pour décrire les BM associés aux projets open source. Un BM est clairement lié au libre : il s'agit de l'engagement. Les trois autres se situent dans l'open source. Il s'agit des BM de l'exploration, de l'expertise et de l'optimisation.

Dans la plupart des cas, un éditeur génère un projet unique et la description de son BM permet de comprendre comment l'éditeur choisit de développer son activité et d'en retirer des bénéfices. Parfois, les éditeurs portent plusieurs projets et dans ce cas, ils peuvent gérer un portefeuille de projets aux BM potentiellement différents. Dans tous les cas, la réflexion sur les BM amène à concilier une approche empirique - car, il s'agit de bien décrire pratiquement comment le processus se réalise - à une approche stratégique et philosophique. Le modèle open source crée des incertitudes. Les codes sont ouverts, les contributions sont libres, la création de valeur est aléatoire, les modes d'appropriation sont divers, voire obscurs. Dans ce contexte, il est difficile d'anticiper la valeur créée et le partage de la valeur. Les contributeurs identifient des signaux tels que l'affiliation ou les droits de propriété pour décider de leur engagement. Finalement, l'anticipation de l'appropriation, si elle n'est pas clairement dite et formalisée intervient et influence les contributions et donc la création de valeur elle-même. Dans ce contexte, s'il est important de comprendre les intérêts que peuvent avoir les acteurs à contribuer à un processus nouveau, il faut aussi que les valeurs fondatrices soient effectivement respectées pour que le système fonctionne. 


\section{BIBLIOGRAPHIE}

Arthur, W.B. (1989), « Competing Technologies, Increasing Returns and Lock-In by Historical Events », Economic Journal, vol. 99, n³94, p.116-131.

Baden-Fuller, C., et Morgan, M.S. (2010), « Business Models as Models », Long Range Planning, vol. $43, n^{\circ} 2-3$, p. 156-171.

Benkeltoum, N. (2011), Gérer et comprendre l'open source: Une modélisation en termes de "régimes », Presses des Mines.

Bonaccorsi, A., Giannangeli, S., et Rossi, C. (2006), « Entry Strategies Under Competing Standards: Hybrid Business Models in the Open Source Software Industry », Management Science, vol. 52, $\mathrm{n}^{\circ} 7$, p. 1085-1098.

Borgès Da Silva, R. (2013), « Taxonomie et typologie : est-ce vraiment des synonymes ? ", Santé Publique, vol. 25, $\mathrm{n}^{\circ}$ 5, p. 633-637.

Boudreau, K.J., et Lakhani, K.R. (2009), « How to Manage Outside Innovation », MIT Sloan Management Review, vol. 50, n 4, p. 69-76.

Casadesus-Masanell, R., et Tarziján, J. (2012), « When One Business Model Isn’t Enough », Harvard Business Review, vol. 90, January-February, p. 132-137.

Chesbrough, H. (2003), Open Innovation: The New Imperative for Creating and Profiting from Technology , Harvard Business School Press.

Chesbrough, H. (2006), « A Framework for Advancing Your Business Model », in H. Chesbrough, Open Business Models, Harvard Business School Press, p. 107-134.

Chesbrough, H. (2010), « Business Model Innovation: Opportunities and Barriers », Long Range Planning, vol. 43, n 2-3, p. 354-363.

Chesbrough, H., et Rosenbloom, R.S. (2002), « The Role of the Business Model in Capturing Value from Innovation: Evidence from Xerox Corporation's Technology Spin-off Companies ", Industrial and Corporate Change, vol. 11, $\mathrm{n}^{\circ} 3$, p. 529-555.

Chesbrough, H., Vanhaverbeke, W., et West, J. (2006), Open Innovation: Researching a New Paradigm, Oxford University Press.

Demil, B., et Lecocq, X. (2003), « Comment exploiter brevets et marques », L'Expansion Management Review, Juin, $\mathrm{n}^{\circ}$ 109, p. 88-95.

Demil, B., et Lecocq, X. (2006), « Neither Market nor Hierarchy or Network: The Emerging Bazaar Governance », Organization Studies, vol. 27, n 10, p. 1447-1466.

Ghaziani, A., et Ventresca, M.J. (2005), « Keywords and Cultural Change : Frame Analysis of Business Model Public Talk 1975-2000», Sociological Forum, vol. 20, n 4, p. 523-559.

Johnson, M.W., Christensen, C., et Kadermann, H. (2008), « Reinventing Business Model », Harvard Business Review, vol. 86, $\mathrm{n}^{\circ}$ 12, p. 59-68.

Lecocq, X., Demil, B., et Ventura, J. (2010), « Business Models as a Research Program in Strategic Management : An Appraisal Based on Lakatos », Management, vol. 13, n 4, p. 214-225. 
Lieberman, M., et Montgomery, D. (1988), « First-mover Advantages », Strategic Management Journal, vol. 9, n S1, p. 41-58.

Magretta, J. (2002), « Why Business Models Matter », Harvard Business Review, vol. 80, May, p. 3-8. Malhotra, A., et Majchrzak, A. (2014), « Managing Crowds in Innovation Challenges », California Management Review, vol. 56, $\mathrm{n}^{\circ} 4$.

Mouakhar, K., et Tellier, A. (2013), « Comment concilier marchand et non-marchand : une taxonomie empirique des comportements stratégiques des SSLL », Systèmes d'Information et Management, vol. 18, $\mathrm{n}^{\circ}$ 3, p. 1-34.

Muselli, L. (2008), « Le rôle des licences dans les modèles économiques des éditeurs de logiciels open source ", Revue Française de Gestion, vol. 34, n 181, p. 199-214.

Okazaki, S. (2006), « What Do We Know About Mobile Internet Adopters? A cluster Analysis », Information \& Management, vol. 42, $\mathrm{n}^{\circ} 2$.

Pénin, J. (2011), « Open Source Innovation : Towards a Generalization of the Open Source Model Beyond Software ", Revue d'Économie Industrielle, vol. 136, $4^{\mathrm{e}}$ trimestre.

Porter, M.E. (2001), « Strategy and the Internet », Harvard Business Review, vol. 79, n 3, p. 62-78.

Rajala, R., Rossi, M., et Tuunainen, V.K. (2003), « A Framework for Analyzing Software Business Models ", $11^{\text {th }}$ European Conference on Information Systems, Naples, Italy.

Teece, D.J. (2010), « Business Models, Business Strategy and Innovation », Long Range Planning, vol. $43, \mathrm{n}^{\circ} 2-3, \mathrm{p} .172-194$.

Tkaczynski, A. (2017), Segmentation Using Two-Step Cluster Analysis, in T. Dietrich, S. Rundle-Thiele, et K. Kubacki (Eds.), Segmentation in Social Marketing: Process, Methods and Application, Springer, p. 109-125.

West, J., Salter, A., Vanhaverbeke, W., et Chesbrough, H. (2014), « Open Innovation: The Next Decade ", Research Policy, vol. 43, n 5, p. 805-811.

Williamson, O. E. (1996), The Mecanisms of Governance, Oxford University Press.

Wirtz, B.W., Pistoia, A., Ullrich, S., et Göttel, V. (2016), « Business Models: Origin, Development and Future Research Perspectives ", Long Range Planning, vol. 49, n 1, p. 36-54.

Wirtz, B. W., Schilke, O., et Ullrich, S. (2010), « Strategic Development of Business Models », Long Range Planning, vol. 43, p. 272-290.

Zott, C., et Amit, R. (2010), « Business Model Design: An Activity System Perspective », Long Range Planning, vol. 43, n 2-3, p. 216-226.

Zott, C., Amit, R., et Massa, L. (2010), « The Business Model: Theoretical Roots, Recent Developments, and Future Research », IESE Working Papers, DI-862-E.

Zott, C., Amit, R., et Massa, L. (2011), « The Business Model: Recent Developments and Future Research », Journal of Management, vol. 37, n4, p. 1019-1042.

\section{NOTES}

1. https://octoverse.github.com/

1. Étude Pierre Audoin Consultants 2015

1. Signification asymptotique inférieure à 0,05 


\section{RÉSUMÉS}

Cette recherche identifie les business models (BM) mis en œuvre par les éditeurs de logiciels libres et open source. Ces modèles requièrent une approche originale des BM parce que la création de la valeur dépend de l'attractivité du projet auprès de contributeurs dont le nombre, la qualité et la diversité ne sont pas contrôlés. Cette spécificité pose la question du partage d'une valeur qui ne peut pas être anticipée ni formellement négociée. Nous procédons à une analyse quantitative de près de 200 logiciels et réalisons une taxonomie par la méthode TwoStep Cluster. Nos résultats mettent au jour quatre BM, engagement, exploration, expertise et optimisation.

This research identifies business models (BM) adopted by editors of free and open source software. These models require an original BM approach because the value creation depends on the project attractiveness on contributors whose number, quality and diversity are not controlled. This specificity raises the question of how sharing a value that cannot be anticipated or formally negotiated. We carry out a quantitative analysis of nearly 200 software and perform a taxonomy using the TwoStep Cluster method. Our results reveal four BMs, commitment, exploration, expertise and optimization.

\section{INDEX}

Mots-clés : business model ; open source ; innovation ouverte

Keywords : business model; open source; open innovation

jel L17 Open Source Products and Markets, L21 - Business Objectives of the Firm

\section{AUTEURS}

\section{AMEL CHARLEUX}

Doctorante, Université de Montpellier

Montpellier Recherche en Management

\section{ANNE MIONE}

Professeur, Université de Montpellier

Montpellier Recherche en Management 